\title{
Evaluation of the Medical Image Compression using Wavelet Packet Transform and SPIHT Coding
}

\author{
Ismahane Benyahia, Mohammed Beladgham, Abdesselam Bassou \\ LTIT Laboratory, Tahri Mohammed University of Bechar, Bechar, Algeria
}

\begin{tabular}{l}
\hline \hline Article Info \\
\hline Article history: \\
Received Oct 1, 2017 \\
Revised Jan 16, 2018 \\
Accepted Apr 1, 2018 \\
\hline Keyword: \\
Compression \\
Evaluation parameters \\
Medical image \\
SPIHT \\
Wavelet packets \\
\hline
\end{tabular}

\begin{abstract}
Wavelet transforms and wavelet packets are widely imposed in the analysis and resolution of problems related to science and technical engineering. Decomposition wavelet packet allows several frequency bands according to various levels of resolutions. We apply this transform (PWT) coupled with the SPIHT coder to reduce the limitations of conventional wavelet filter bank. The results obtained using the applied algorithm, are very satisfactory and encouraging compared to many of the best coders cited in the literature and show a visual and numerical superiority over conventional methods. These the promising results are confirmed by visual evaluation parameters (PSNR, MSSIM and VIF).
\end{abstract}

Copyright $\odot 2018$ Institute of Advanced Engineering and Science. All rights reserved.

\section{Corresponding Author:}

Abdesselam Bassou,

LTIT Laboratory, Department of Electrical Engineering,

Tahri Mohammed University of Bechar,

P.O. Box 417, 08000, Bechar, Algeria.

Email: a.bassou@gmail.com

\section{INTRODUCTION}

In the medical field, the image is nowadays an indispensable support for the diagnosis, the establishment of treatment protocols, patient monitoring, and operative preparation. These images must be retained for a certain period, the storage required for archiving all data is constantly changing. The images are often consulted locally, but they can also be remotely through networks with bandwidth limited. The use of compression so quickly revealed to be essential, both to facilitate storage or remote browsing in these masses of data [1].

The wavelet packet decomposition is an extension of the transforms discrete wavelet to select the paving of the time-frequency plane. This choice is made through the selection of a base wavelet packet .In general; the base is selected according to the processed signal according to a criterion satisfying the constraints application. This database will be called the best basis [2]. Our objective is based on a comparative study between the different images types to which it will apply the wavelet packet transform with progressive encoder SPHIT. The estimation and judgment of the compressed image quality is given by the evaluation parameters (PSNR, MSSIM and FIV).

\section{WAVELET PACKET}

The decomposition of the signal wavelet packet is a generalization of multi-resolution analysis. Spaces approximations are always cut in the same way the details of spaces too are direct sum of two sub inferior temporal resolutions space (divided by 2). The packets thus wavelet decomposition of a signal is an extension of the transforms discrete wavelet [3]. Figure 1 represents the pyramidal algorithm extended for obtaining the coefficients. As for the fast calculation of the coefficients of the wavelet transform of a signal, 
one proceeds by successive filtering and decimation of the signal. Here, the detail coefficients are also decomposed.

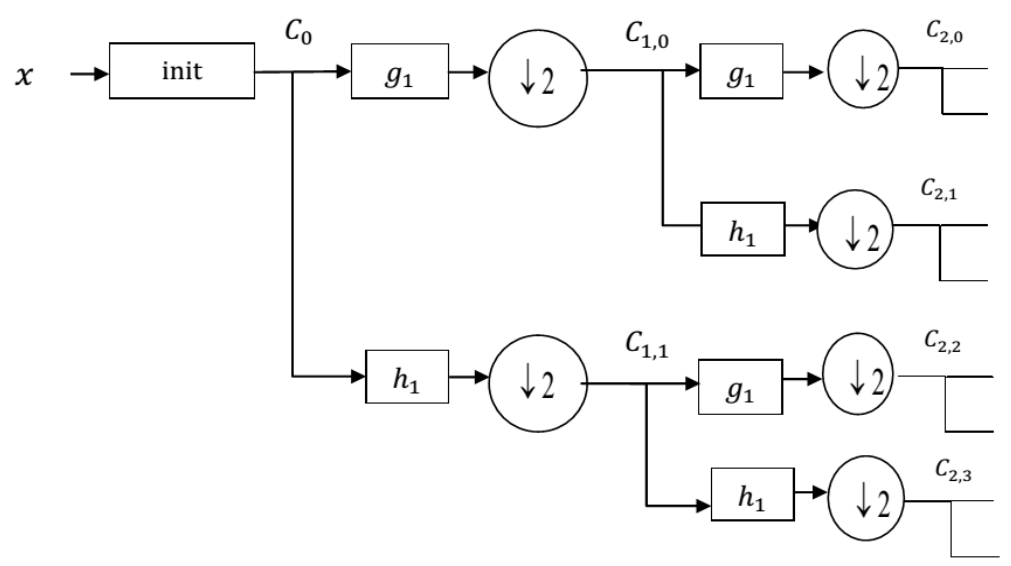

Figure 1. Scheme of the decomposition algorithm wavelet packet of a signal

$\mathrm{g}_{1}$ and $\mathrm{h}_{1}$ are the quadratic filter mirror QMF filters associated with the scale $\phi$ and wavelet $\psi$ functions. $C_{i, j}$ are called wavelet packets. Each packet $C_{i, j}$ contains $n / j^{-2}$ coefficients in the framework of the decomposition of a signal of length $n$. Coefficients of wavelet packets are rated $C_{i, m}(k)$, where $\mathbf{j}$ is the level of resolution, $\mathbf{m}$ is the spectral band and $\mathbf{k}$ is the index of translation. They are obtained by the decomposition of the signal on the bases generated by $\mathrm{W}_{\mathrm{m}}$ functions [4]:

$$
\begin{aligned}
& \mathrm{C}_{\mathrm{j}, \mathrm{m}}(\mathrm{k})=\left\langle\mathrm{x}(\mathrm{t}), 2^{-\mathrm{j} / 2} \mathrm{~W}_{\mathrm{m}}\left(2^{-\mathrm{j}} \mathrm{t}-\mathrm{k}\right)\right\rangle \\
& \mathrm{W}_{2 \mathrm{~m}(\mathrm{t})}=2^{1 / 2} \sum_{\mathrm{k}} \mathrm{g}_{\mathrm{k}} \mathrm{W}_{\mathrm{m}}(2 \mathrm{t}-\mathrm{k}) \\
& \mathrm{W}_{2 \mathrm{~m}+1(\mathrm{t})}=2^{1 / 2} \quad \sum_{\mathrm{k}} \mathrm{h}_{\mathrm{k}} \mathrm{W}_{\mathrm{m}}(2 \mathrm{t}-\mathrm{k})
\end{aligned}
$$

where, $W_{0}$ corresponds to the $\phi$ function, and $W_{1}$ corresponds to the $\psi$ function

\subsection{Redundancy of information}

Figure 2 shows the binary tree decomposition of wavelet packets to one of a signal size.

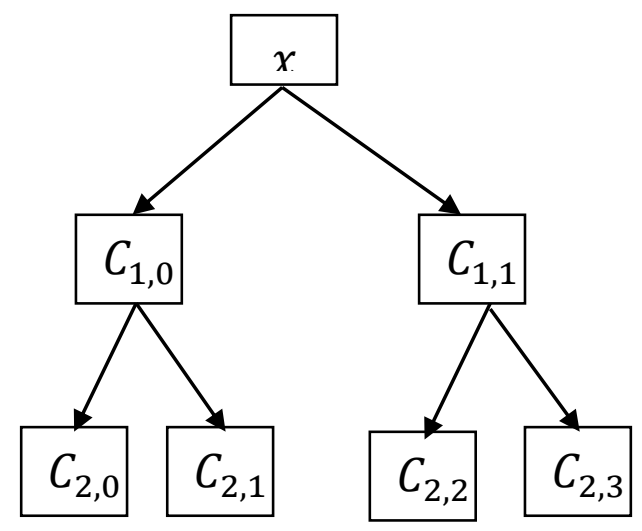

Figure 2. Binary tree decomposition of a signal into wavelet packets

At each level of the tree, all the signal information is shown. At a given level of decomposition, corresponds to a regular frequency division. At the last level, each frequency is represented by a point, we 
lost all the temporary information then we have purely frequency decomposition of the signal. Figure 3 shows the paving of the time-frequency plane for a signal $x \in R^{8}$ for each level of the tree [5].
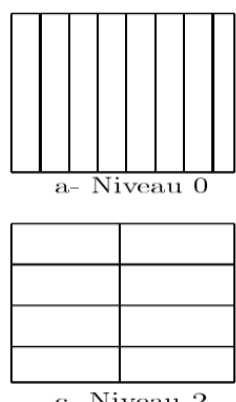

c- Niveau 2
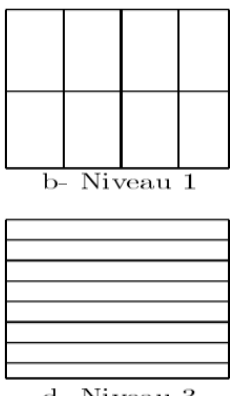

d- Niveau 3

Figure 3. Paving time frequencies corresponding to each level of the decomposition tree

\section{WAVELET PACKET BASE}

Binary tree decomposition wavelet packet thus provides a highly redundant representation of the signal. If one wants to work with a non-redundant representation, he has to choose a packet-based that is to say a set of nodes in the tree whose projection in the time-frequency space forms a partition. This is derived from the notion of eligible tree. A qualified tree is composed of nodes with 0 or 2 strings; the base will consist of all nodes with no string. Considering the frequency division induced decomposition wavelet packet this goes back to cover the frequency axis without overlapping.
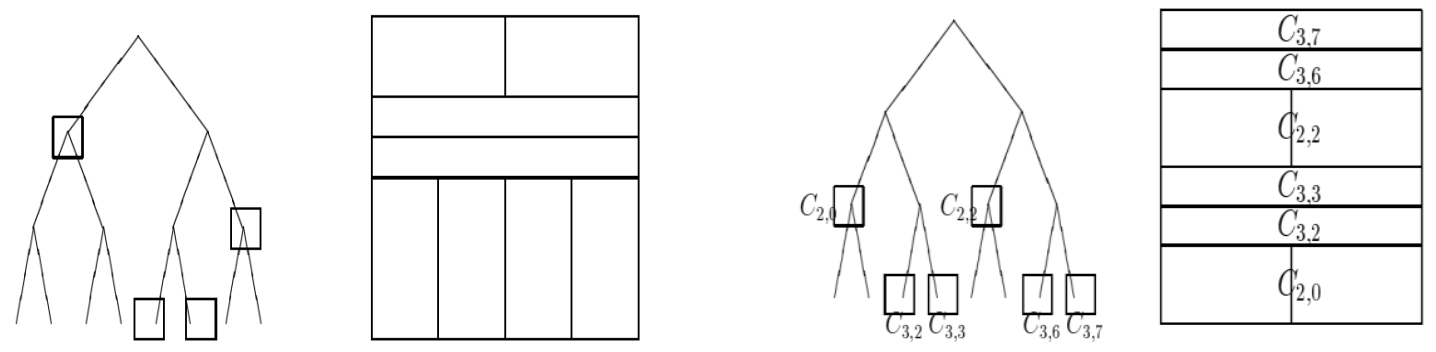

Figure 4. Examples of wavelet packet bases and paving time frequencies corresponding

Figure 4 shows two examples of wavelet packet bases. The nodes selected in the basic constitution are surrounded by squares. We see that if we prune the trees at the selected nodes, eligible trees are obtained. The selected packages can form a partition of the frequency axis (horizontal on the tree). The paving of the time-frequency induced by the foundation is shown for a signal $x \in \mathrm{R}^{8}$ [6].

The packet-based wavelet decomposition from the principle of the AMR, we can reconstruct the signal using reconstruction filters associated with decomposition filters. If $\mathrm{W}_{\mathrm{m}}$ is the dual basis functions associated with $W_{m}$, and $B$ is the set of indices $\{j, m\}$ selected nodes in a packet wavelet basis [4], then

$$
\mathrm{x}_{(\mathrm{t})}=\sum_{\{\mathrm{j}, \mathrm{m}\} \in \mathrm{B}} \quad \sum_{\mathrm{k}} \mathrm{C}_{\mathrm{i}, \mathrm{m}}(\mathrm{k}) \frac{1}{2^{\mathrm{j} / 2}} \mathrm{~W}_{\mathrm{m}}\left(2^{-\mathrm{j}} \mathrm{t}-\mathrm{k}\right)
$$

\section{GENERALIZATION TO IMAGES}

The decomposition of an image on a wavelet packet base follows the same principle as the decomposition of a one-dimensional signal. We generalize the wavelet decomposition algorithm filtering and decimating coefficients details and approximations. In the case of images are act on the lines and columns, it will then quadtree decomposition wavelet packets represented by the Figure 5.

Representation in the Quaternary wavelet packet, each packet $C_{p, i, j}$ corresponding to a node of the tree contains information regarding the whole image in the frequency band indexed by (i; $\mathrm{j}$ ) and whose size is determined by the resolution level $\mathrm{P}$. The coefficients of the packet are rated $\mathrm{C}_{\mathrm{p}, \mathrm{i}, \mathrm{j}}(\mathrm{k}, \mathrm{l})$. This decomposition

Evaluation of the Medical Image Compression using Wavelet Packet Transform ... (Ismahane Benyahia) 
can be interpreted as the decomposition into sub-bands of the image, with increasing frequency resolutions [7].

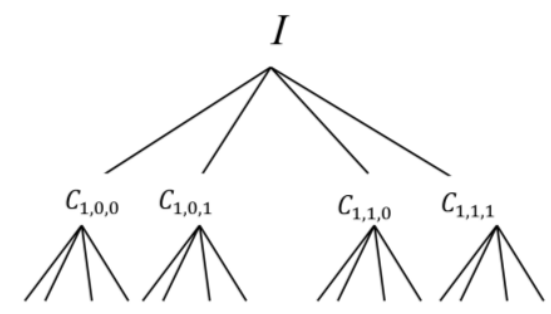

Figure 5.Quadtree decomposition wavelet packet

\section{THE ENCODING ALGORITHM SPIHT (SET PARTITIONING IN HIERARCHICAL TREE)}

The SPIHT algorithm [8] involves a list of insignificant sets (LSP), a list of insignificant coefficients (LIS) and a list of significant coefficients (LIP). In this work we applied the modified SPIHT algorithm which is the most adapted with wavelet packets [9]. The modified SPIHT has the same principle as the SPIHT encoder; so for easier representation, the entire sub-band is treated as a node in a wavelet tree. The decomposition level of a certain sub-band is the same as that of the dyadic wavelet decomposition subband from which it is obtained by further decomposition (i.e. the same level does not imply the same scale - just the same position on a dyadic wavelet tree).

The natural parent (NP) is a subband whose child is on the next finer scale and after lower wavelet decomposition tree level. In addition, a NP's child must have the same relative position as NP, if a relative subband position is defined for the dyadic subband from which it is obtained by further decomposition. For example, each of the children of T3 in Figure 6 has a child who is in the same relative position. And just to be mentioned, all parents in dyadic wavelet decompositions are natural. With regular zerotrees, as in SPIHT, each parent coefficient has four of its children on the same spatial location and immediately at the next lower level of the tree. In wavelet decomposition trees, the parent-child allocation is complicated by the fact that there may be different scale differences between the subbands on the adjacent levels [10].

Four different cases of inter-level dependencies are organized. All subbands of the next lower level are called child subbands here, although when parental conflicts are resolved, the true parent can be found in some higher level.

a. Subband has within the same relative position child subbands which are on a coarser scale, as it occurred in the subtree of $\mathrm{T} 2$.

b. Subband has within the same relative position child subband that is on the same scale, as it occurred in the subtree of $\mathrm{T} 2$.

c. Natural parent-child relation - subband has within the same relative position child subbandthat is on a next finer scale, as it occurred in the subtree of T3.

d. Subband's relative position overlaps with the relative position of a child subband that is on a scale more than one level finer, as it occurred in the subtree of T3.
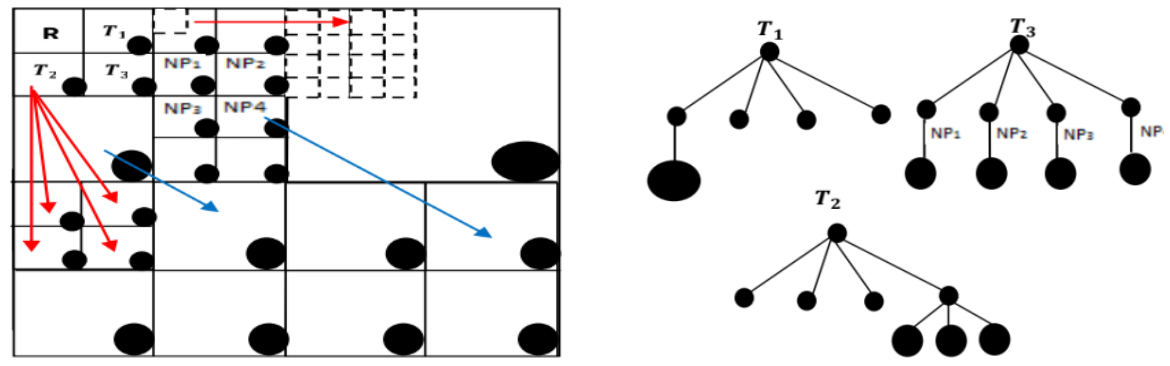

Figure 6. SPIHT packet wavelet algorithm 


\section{EVALUATION PARAMETERS OF THE QUALITY}

The commonly parameter used in image compression is the mean square error MSE. This variable (error) is defined by the mean square between the pixel $(i, j)$ of the original image $I(i, j)$, and the pixel $(i, j)$ of the reconstructed image $\hat{I}(i, j)[11],[12]$.

$$
M S E=\frac{1}{M \cdot N} \sum_{m=0}^{M-1} \sum_{n=0}^{N-1}[I(i, j)-\hat{I}(i, j)]^{2}
$$

The peak signal to noise ratio is given by [13], [14]:

$$
P S N R=10 \cdot \log _{10} \frac{\left(2^{R}-1\right)^{2}}{M S E}[d B]
$$

We then evaluate a new paradigm for assessing the quality of medical images, the similarity index compares the brightness, contrast, and structure between each pair of vectors, where the index of structural similarity (SSIM) between two signals $\mathrm{x}$ and $\mathrm{y}$ is given by the following expression:

$$
\operatorname{SSIM}(x, y)=l(x, y) \cdot c(x, y) \cdot s(x, y)
$$

Finally, we need a single global measure of overall image quality that is given by the formula [15], [16]:

$$
\operatorname{MSSIM}(I, \hat{I})=\frac{1}{M} \sum_{i=1}^{M} \operatorname{SSIM}\left(I_{i}, \hat{I}_{i}\right)
$$

$\mathrm{M}$ is the total number of local windows in the image. The values MSSIM exhibit greater consistency with the visual quality [17]. The Visual Information fidelity parameter (VIF) quantifies Shannon information that is shared between the reference and distorted images with respect to the information contained in the reference image itself [18]. VIF test is then evaluated as:

$$
\mathrm{VIF}=\frac{\sum_{j}^{\mathrm{M}} \mathrm{I}\left(\mathrm{C}^{\mathrm{j}} ; \frac{\mathrm{F}^{\mathrm{j}}}{\mathrm{j}^{\mathrm{j}}}\right)}{\sum_{\mathrm{j}}^{\mathrm{M}} \mathrm{I}\left(\mathrm{C}^{\mathrm{j}} ; \frac{\mathrm{E}^{\mathrm{j}}}{\mathrm{s}^{\mathrm{j}}}\right)}
$$

where, $\mathrm{I}(\mathrm{X} ; \mathrm{Y} / \mathrm{Z})$ is the conditional mutual information between $\mathrm{X}$ and $\mathrm{Y}$, conditioned to $\mathrm{Z} ; \mathrm{s}^{\mathrm{j}}$ is a realization of $S^{j}$ for a particular image, the index $\mathrm{j}$ runs through all sub-bands in the decomposed image [19].

\section{RESULTS AND ANALYSIS}

In this work, we are interested in the compression of medical image by the transformed wavelet packet (PWT) coupled to the coder SPIHT. For this, we have a MRI image of a brain (axial) [20], of size $512 \times 512$ (grayscale) encoded on 8 bpp recorded through an MRI scanner. We vary the bit rate (Rc) of 0.125 to $2 \mathrm{bpp}$ and calculate the evaluation parameters Simulation results of the applied algorithm is illustrated in the Figure 7. From Figure 7 we note that the applied algorithm provides important values of PSNR, MSSIM and VIF for a bit rate of $0.5 \mathrm{bpp}$.

To better demonstrate the effectiveness and performance of applying PWT in the medical field, we will now make a comparison between different types of transformed DWT and PWT coupled with the encoder Progressive SPIHT the lifting structure [21], [22]. For this, we chose the same picture MRI of the Figure 7 with a level of decomposition $\mathrm{N}=4$ we vary the bit rate from 0.125 to 2 and calculate the evaluation parameters. The results are given in Figure 8.

To illustrate the effectiveness of the applied algorithm we applied different algorithms mentioned previously another IRM2 medical image size $512 \times 512$ (grayscale) encoded on $8 \mathrm{bpp}$. We vary the bit rate of 0.125 to 2 and calculate the assessment parameters. The results obtained are given in Table 1 . We distinguish that from a bit rate of 0.5 bpp we obtain suitable values of PSNR, MSSIM and FIV. 


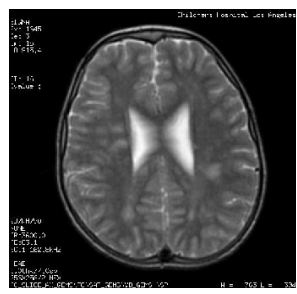

Axial slice of brain (Image original)

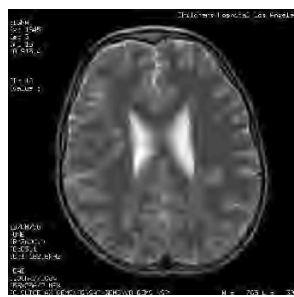

(c) Rc=0.5 bpp; MSSIM $=0.95 ;$ VIF $=0.68$; PSNR $=38.24 \mathrm{~dB}$

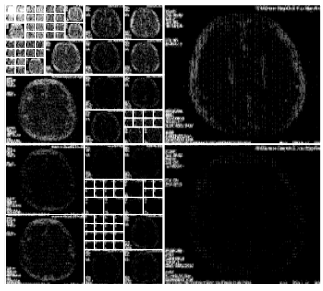

Decomposition by the transform wavelet packets

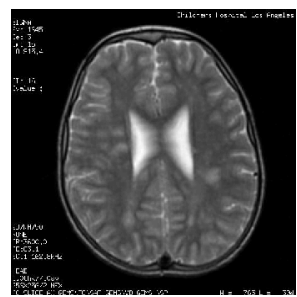

(d) Rc=0.75 bpp; MSSIM=0.98; VIF $=0.80$ $\mathrm{PSNR}=43.14 \mathrm{~dB}$

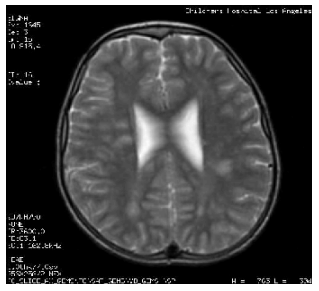

(a) $\mathrm{Rc}=0.125 \mathrm{bpp}$; MSSIM=0.78; VIF = 0.27; PSNR $=24.81 \mathrm{~dB}$

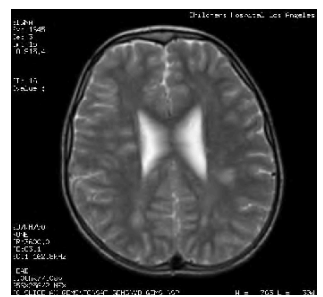

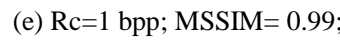
$\mathrm{VIF}=0.88 ;$ PSNR $=46.63 \mathrm{~dB}$

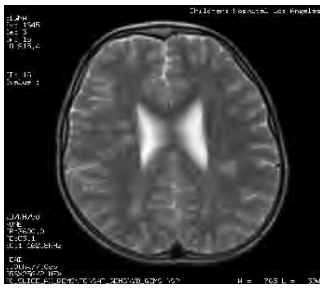

(b) $\mathrm{Rc}=0.25 \mathrm{bpp}$; MSSIM=0.88; VIF $=0.42$; $\mathrm{PSNR}=30.69 \mathrm{~dB}$

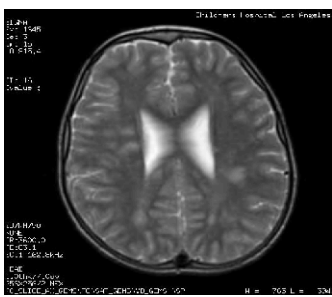

(f) Rc=2 bpp; MSSIM=1; $\mathrm{VIF}=0.98 ; \mathrm{PSNR}=55.19 \mathrm{~dB}$

Figure 7. MRI image compression by CDF9/7 (PWT) + SPIHT
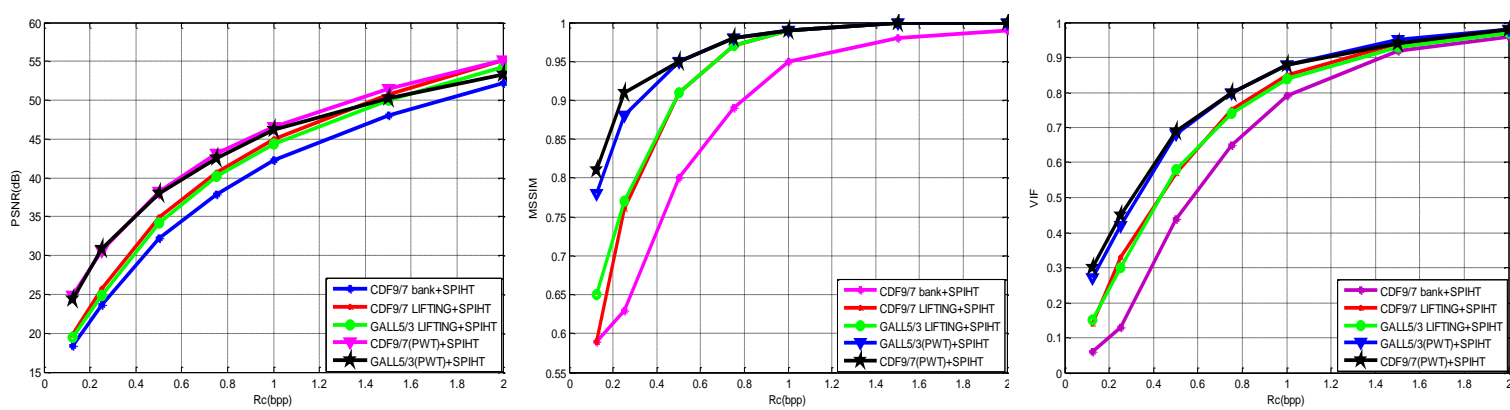

Figure 8. PSNR, MSSIM, and VIF Variation of MRI image

Patient - Male

Age - 52 years

Exam performed with CTL coil

Sag FRFSE-XL T2 4mm

Patient in serious aggravation

Plaque of demyelization, multiple sclerosis [20]

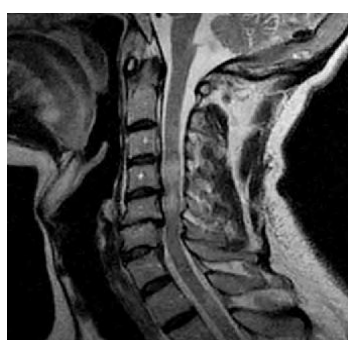

Original image

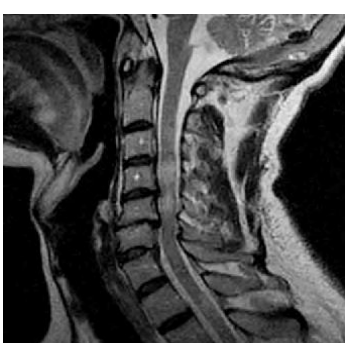

Reconstructed image by CDF 9/7 $(\mathrm{PWT})+$ SPIHT

$\mathrm{PSNR}=38.46 \mathrm{~dB} ; \mathrm{MSSIM}=0.94$; $\mathrm{VIF}=0.70$

Figure 9. MRI2 image compression by CDF9/7 $(\mathrm{PWT})+$ SPIHT $(\mathrm{Rc}=0.5 \mathrm{bpp})$ 
This study was widespread on three medical images (MRI2, TDM and echography) from the medical database GE Medical System [23]. The following Figure 9 and Figure 10 show the results obtained after the application of PWT + SPIHT algorithm for a rate of $0.5 \mathrm{bpp}$. As a result, we can state, from Figure 9 and Figure 10, that from $0.5 \mathrm{bpp}$, the applied algorithm gives an acceptable image quality.

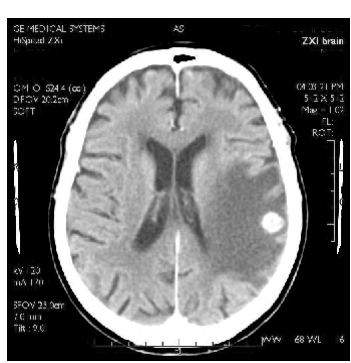

Original image (TDM)

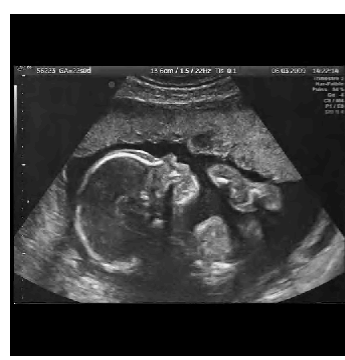

Original image (ECHO)

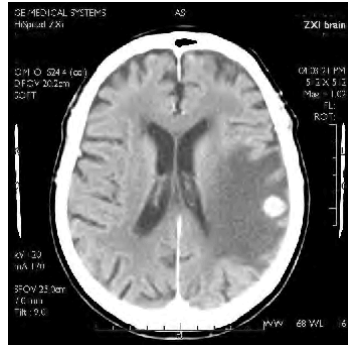

Reconstructed TDM image by CDF9/7(PWT) +SPIHT PSNR $=33.20 \mathrm{~dB}, \quad$ MSSIM $=$ $0.89 ; \mathrm{VIF}=0.55$

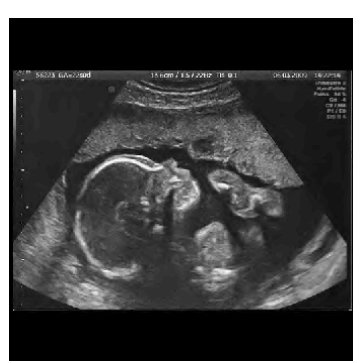

Reconstructed ECHO image by CDF9/7(PWT) +SPIHT

$\mathrm{PSNR}=34.43 \mathrm{~dB}$ $\mathrm{MSSIM}=0.90 ; \mathrm{VIF}=0.50$

Figure 10. TDM and ECHO medical images compression by CDF9/7 (PWT) + SPIHT algorithm $(\mathrm{Rc}=0.5 \mathrm{bpp})$

Table 1. PSNR, MSSIM, and VIF Variation of IRM2 medical image

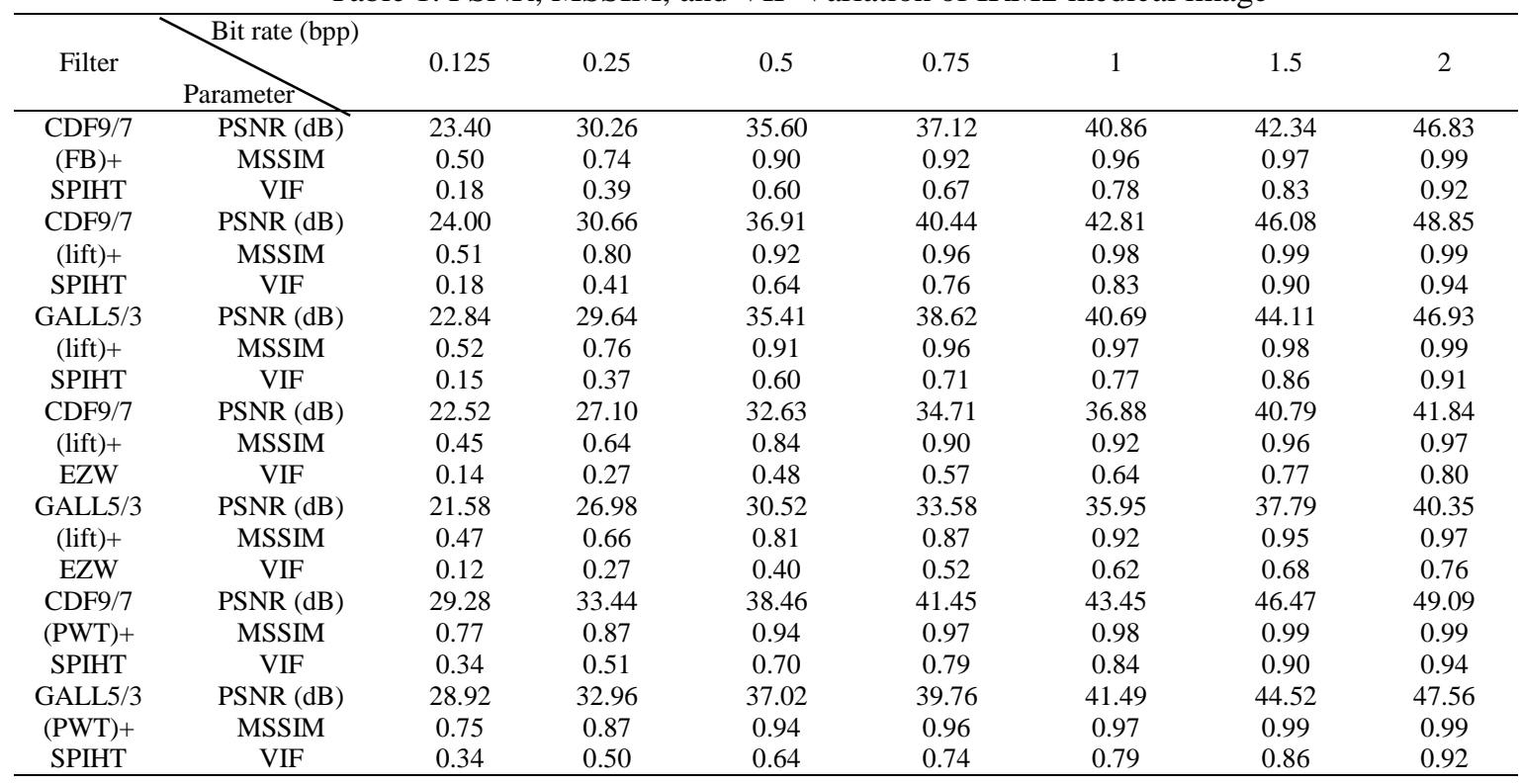

\section{CONCLUSION}

In this paper, we applied a compression approach on medical images using wavelet packet coupled with the progressive coder SPIHT. This approach was tested on medical images (MRI, TDM and ECHO), the results are satisfactory. Interesting compression ratio and good quality of the reconstructed images are obtained in comparison with other algorithms (DWT, LIFTING). The applied method allows to preserve fine structures and produces images with better quality, which is important for medical applications and this is proved by the important values of the evaluation parameters.

\section{ACKNOWLEDGEMENTS}

The authors want to thank the members and the directors of LTIT Laboratory of Tahri Mohammed university of Bechar for their great availability, their encouragement, and their precious advices. We would also like to thank the Editor and anonymous reviewers for their comments and suggestions. 


\section{REFERENCES}

[1] J. Taquet, "Advanced techniques for the compression of medical images," PhD Thesis, University of Rennes 1, France, 2011.

[2] A. Manoury, "Tattooing of wavelet packet digital images," PhD thesis, University of Nantes, France, 2001.

[3] E. Hitti, "Selection of an optimal bank of filters from a decomposition into wavelet packets. Application to frequency jumpg detection in multicomponent signals," PhD thesis, University of Nantes, France, 1999.

[4] G. Strang, "Wavelet and filter bank," Wellesley Cambridge Press, 1996.

[5] A. Muntasa, I. A. Sirajudin, M. H. Purnomo, "Appearance global and local structure fusion for face image recognition”, TELKOMNIKA (Telecommunication, Computing, Electronics and Control), 2011, vol. 9, no. 1, pp. $125-132$.

[6] A. Hazarathaiah and B. P. Rao, "Medical Image Compression using Lifting based New Wavelet Transforms," International Journal of Electrical and Computer Engineering (IJECE), vol. 4, no. 5, pp. 741-750, 2014.

[7] A. Zitouni, "Wavelets and Digital Image Compression Techniques," PhD Thesis, University Mohamed Khider Biskra, Algeria, 2013.

[8] S. NirmalRaj, "SPIHT: A Set Partitioning in Hierarchical Trees Algorithm for Image Compression," Contemporary Engineering Sciences, vol. 8, no. 6, pp. 263-270, 2015.

[9] M. Beladgham, et al., "Improving Quality of Medical Image Compression Using Biorthogonal CDF Wavelet Based on Lifting Scheme and SPIHT Coding," Serbian journal of electrical engineering, vol. 8, no. 2, pp. 163-179, 2011.

[10] N. Sprljan, et al., "Modified Spiht Algorithm For Wavelet Packet Image Coding," IEEE International Symposium on Video \& image Processing and Multimedia Communications, Croatia, pp. 16-19, 2002.

[11] C. J. Renoh, et al., "Curvelet Transform based Retinal Image Analysis," International Journal of Electrical and Computer Engineering (IJECE), vol. 3, no. 3, pp. 366-371, 2013.

[12] M. Beladgham, et al., "A Comparative Study between Bandelet and Wavelet Transform Coupled by EZW and SPIHT Coder for Image Compression," International Journal of Image, Graphics and Signal Processing (IJIGSP), vol. 5, no. 12, 2013.

[13] M. Beladgham, et al., "Medical Image Compression Using Quincunx Wavelets and SPIHT Coding," Journal of Electrical Engineering \& Technology, vol. 7, no. 2, pp. 264-272, 2012.

[14] L. Zhang, et al., "FSIM: A feature similarity index for image quality assessment," IEEE Transactions on Image Processing, vol. 20, pp. 2378-2386, 2011.

[15] C. J. Renoh, et al., "Curvelet Transform based Retinal Image Analysis," International Journal of Electrical and Computer Engineering (IJECE), vol. 3, no. 3, pp. 366-371, 2013.

[16] E. Dumic, et al., "New image-quality measure based on wavelets," Journal of Electronic Imaging, vol. 19, no. 1, pp. 11-18, 2010.

[17] Y. Habchi, et al., "RGB Medical Video Compression Using Geometric Wavelet," International Journal of Electrical and Computer Engineering (IJECE), vol. 6, no. 4, pp. 1627-1636, 2016.

[18] A. K. Moorthy, et al., "Visual Perception and Quality assessment," Optical and digital image processing, Chap. 19, Wiley, 2010.

[19] Z. Wang and Q. LI, "Information Content Weighting for Perceptual Image Quality Assessment," IEEE Transactions on Image Processing, vol. 20, no. 5, pp. 1185-1198, 2011.

[20] www.gehealthcare.com (database).

[21] M. Beladgham, et al., "MRI image compression using biorthogonal CDF wavelet based on lifting scheme and SPIHT coding," International Conference on Electrical Engineering (CIGE'10), Proceedings of Journal of Scientific Research, vol. 2, pp. 225-232, 2010.

[22] M. Beladgham, et al., "New Contribution on Compression Color Images: Analysis and Synthesis for Telemedicine Applications," International Journal of Information Engineering and Electronic Business (IJIEEB), vol. 6, no. 2, pp. 28-34, 2014.

[23] www.gemedicalsystem.com (database).

\section{BIOGRAPHIES OF AUTHORS}

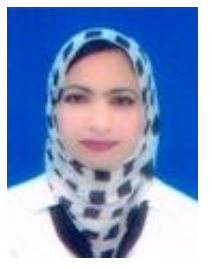

Ismahane Benyahia was born in Bechar, Algeria. She received the dipl. El-Ing from the University of Bechar, Algeria, in 2009, the Master degree in signals and telecommunication from University of Bechar, Algeria in 2014. At present, she prepares the doctoral degree Es-science at University of Bechar, Algeria. Her main research areas are image and video processing, $1 \mathrm{G}$ and $2 \mathrm{G}$ wavelets transform. 


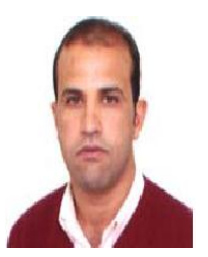

Mohammed Beladgham was born in Tlemcen, Algeria. He received the electrical engineering diploma from university of Tlemcen, Algeria, and then a Master in signals and systems from University of Tlemcen, Algeria and the $\mathrm{PhD}$. degree in Electronics from the University of Tlemcen (Algeria), in 2012. His research interests are Image processing, Medical image compression, wavelets transform and optimal encoder.

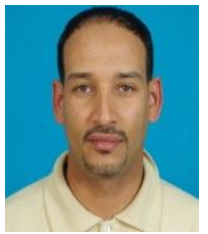

Abdesselam Bassou was born in Bechar, Algeria. He received the Dipl.El.-Ing. Degree from the University of Tlemcen, Algeria in 1997, his Master from the University of Sidi Bel Abbes, Algeria in 2000, and his Doctoral degree Es-Science from the University of Sidi Bel Abbes, Algeria in 2006. Actually, $\mathrm{He}$ is a professor at the University of Bechar, Algeria. His main interests are digital signal processing, turbo encoding schemes and iterative decoding over fading channels, and channel equalization. 